\title{
Nash-Peering: A New Techno-Economic Framework for Internet Interconnections
}

\author{
Doron Zarchy \\ Hebrew University of \\ Jerusalem \\ doronz@cs.huji.ac.il \\ Constantine Dovrolis \\ Georgia Tech \\ constantine@gatech.edu
}

\author{
Amogh Dhamdhere \\ CAIDA \\ amogh@caida.org \\ Michael Schapira \\ Hebrew University of \\ Jerusalem \\ schapiram@huji.ac.il
}

\begin{abstract}
The current framework of Internet interconnections, based on transit and settlement-free peering relations, has systemic problems that often cause peering disputes. We propose a new techno-economic interconnection framework called Nash-Peering, which is based on the principles of Nash Bargaining in game theory and economics. Nash-Peering constitutes a radical departure from current interconnection practices, providing a broader and more economically efficient set of interdomain relations. In particular, the direction of payment is not determined by the direction of traffic or by rigid customer-provider relationships but based on which AS benefits more from the interconnection. We argue that NashPeering can address the root cause of various types of peering disputes.
\end{abstract}

\section{INTRODUCTION}

The tussle between content and access providers has led to interest from techologists and policy makers in the bargaining, money flow, and market power issues behind Internet interconnections [12]. Peering disputes over traffic imbalances are not new - several such incidents between large ISPs and content providers have happened over the years [1]. More recently however, such disputes have been fueled by exploding demand for streaming video, and growing concentration of content among a few providers and CDNs, raising questions about appropriate network management, interconnection business strategies, and the impact of these peering disputes on end-user performance [7].

An important point about the aforementioned peering disputes is that, while they result in performance degradation, they are mostly economic issues. The technical solutions are straightforward - increase the capacity of the network (or shift traffic to other routes) as demand increases. The key questions, however, relate to which party should pay for infrastructure upgrades and how the costs and benefits of interdomain relation- ships should be split among the different parties.

The current interconnection framework is predominantly based on two types of bilateral relations between Autonomous Systems (ASes): transit and settlementfree Peering (sf-Peering) [14]. In transit relations, the provider advertises global Internet routes to the customer, while the latter pays based on the traffic that it sends/receives. In sf-Peering, there is no exchange of money and each AS only exports routes that originate from itself and from its customer-cone. The conditions for establishing an sf-Peering relation depend on the business profile of the corresponding ASes. For instance, content providers tend to peer openly (to minimize their transit fees), while transit providers are more selective, aiming to not peer with ASes that may become their customers in the future [18]. Other types of relations include paid-peering (similar to sf-Peering but one of the two peers gets a traffic-dependent payment from the other) and partial transit (where only some routes are advertised from the provider to the customer) [11].

Our main premise is that the currently deployed interconnection framework has fundamental weaknesses and systemic problems that will continue causing peering disputes. One such weakness is that sf-Peering relationships are formed for reasons (e.g., a balanced traffic ratio) that are often only indirectly related to the costs and benefits of the interconnection [27]. Consequently, some interconnections may not be formed even when they can result in both bilateral and Internet-wide benefits. Instead of looking at each peering conflict as an isolated incident, we need to focus on the limitations of the interconnection framework that is generating these disputes.

In this paper, we propose a new interconnection paradigm that we refer to as Nash-Peering because it is based on the principles of Nash Bargaining in game theory and economics. Nash-Peering constitutes a radical depar- 
ture from today's interconnection practices, providing a broader and more economically efficient set of interdomain relations. In particular, a Nash-Peering interconnection is established if and only if it is beneficial for both parties. Additionally, the direction of payment is not determined by the direction of traffic or by rigid customer-provider relationships but based on which AS benefits more from the interconnection. We argue that Nash-Peering can address the root cause of various types of peering disputes. We finally discuss how the Internet can transition to Nash-Peering.

\section{NASH-PEERING}

\subsection{Overview of Nash bargaining}

Alice and Bob negotiate how to split a hidden treasure of value $V$. Each of them has some information about the treasure's location and they can only find it if they agree on how to split $V$. To recover the treasure each of them will have to spend all their savings: Alice has $s_{A}$ and Bob has $s_{B}$. Obviously, they will be interested in the treasure only if $s_{A}+s_{B}<V$. If they agree, each of them will get a share, say $v_{A}$ and $v_{B}$ respectively, with $v_{A}+v_{B}=V$. Otherwise, if they do not reach agreement, they will stay with their savings $s_{A}$ and $s_{B}$; this is their "outside option" (also referred to as disagreement or threat point).

If they reach an agreement, their cumulative benefit or surplus is $\Delta=V-\left(s_{A}+s_{B}\right)$. The Nash Bargaining solution postulates that Alice should get her outside option $s_{A}$ plus half the surplus; the other half should go to Bob. In other words, Alice should get $v_{A}=s_{A}+$ $\Delta / 2=\left(V+s_{A}-s_{B}\right) / 2$, while Bob should get $v_{B}=$ $s_{B}+\Delta / 2=\left(V+s_{B}-s_{A}\right) / 2$. Note that if Alice, for instance, invests more than Bob $\left(s_{A}>s_{B}\right)$, she should get a larger portion of the treasure, which is arguably a more fair allocation than just splitting $V$ in two equal shares.

Nash showed in 1950 that the previous allocation follows mathematically from a set of reasonable assumptions about the rationality of the two involved individuals [22, 23]; this is an axiomatic theory because it does not derive the Nash solution based on a model of the actual bargaining process. The Nash solution is Pareto-optimal and fair in the sense that it splits the surplus equally between the two players. ${ }^{1}$ In 1986, Binmore, Rubinstein and Wolinsky modeled the bargaining process between two players as a sequential noncooperative game, and they proved, under certain assumptions, that the unique equilibrium is actually the previous Nash solution $\left(v_{A}, v_{B}\right)$ (namely, each player gets her outside option plus half surplus) [3]. An im-

$9^{1}$ There are also other notions of fair bargaining, such as Kalai's solution that maximizes the minimum payoff among the two players. portant condition behind this result is that the risk of breakdown (i.e., the probability that Alice or Bob will walk away from the negotiations even though $s_{A}+s_{B}<$ $V)$ is the same for both players and negligible.

\subsection{Formulation}

Suppose that two ASes, A and B, consider having a direct interconnection between them. To argue quantitatively about the costs and benefits of this interconnection, A and B need to be very specific about the routes and traffic volume that will be going through this interconnection as well as about its location and capacity. Suppose for now that A and B are only negotiating for an interconnection at a specific exchange point at which they are both present, and for a single BGP route that $\mathrm{A}$ will export to $\mathrm{B}$ advertising reachability to a destination prefix D. Suppose that this traffic volume is denoted by $T$. In the rest of this section, all costs and payments refer only to $T$. We will consider the more general case in Section 4.

In the absence of a direct interconnection, flow $T$ is routed through a different network path. For instance, $T$ could flow from $\mathrm{B}$ to a transit provider $\mathrm{C}$ and then to A. This is the "outside option" of A and B. Let $v_{A}^{\prime}$ and $v_{B}^{\prime}$ be the payoffs of $\mathrm{A}$ and $\mathrm{B}$, respectively, when they are not directly interconnected. If $c_{A}^{\prime}$ is the cost incurred by $\mathrm{A}$ for flow $T$ in the outside option, we have that $v_{A}^{\prime}=-c_{A}^{\prime}$; similarly for $\mathrm{B}, v_{B}^{\prime}=-c_{B}^{\prime}$. The costs $c_{A}^{\prime}$ and $c_{B}^{\prime}$ may represent, for instance, transit fees that $\mathrm{A}$ and $\mathrm{B}$ need to pay to their common transit provider C. In a more general case, in which the outside option is associated with both costs and revenues, $c_{A}^{\prime}$ or $c_{B}^{\prime}$ represent the net "costs minus revenues" amount and they may even be negative.

If $\mathrm{A}$ and $\mathrm{B}$ reach an agreement, $T$ will flow directly from $\mathrm{B}$ to $\mathrm{A}$. In general, there will be some $\operatorname{costs} c_{A}$ and $c_{B}$, for $\mathrm{A}$ and $\mathrm{B}$ respectively, associated with this flow. These terms can represent the internal costs of $\mathrm{A}$ and B for carrying this traffic through their own infrastructure.

The surplus of the direct interconnection is

$$
\Delta=\left(c_{A}^{\prime}+c_{B}^{\prime}\right)-\left(c_{A}+c_{B}\right)
$$

Obviously the interconnection makes sense only if the surplus is positive, $\Delta>0$. According to the Nash solution, $\Delta$ should be equally split between $\mathrm{A}$ and $\mathrm{B}$. In the general case, this can only happen if there is a payment between the two players. To see that, consider the case $c_{B}^{\prime}-c_{B}>c_{A}^{\prime}-c_{A}$, i.e., $\mathrm{B}$ benefits from the interconnection more than A. If so, the surplus can be split equally between $\mathrm{A}$ and $\mathrm{B}$ if $\mathrm{B}$ pays a fee $r>0$ to $\mathrm{A}$, such that

$$
r=\frac{\left(c_{B}^{\prime}-c_{B}\right)-\left(c_{A}^{\prime}-c_{A}\right)}{2}
$$

In that case, the benefit that $\mathrm{B}$ gets from the interconnection is equal to the benefit that $\mathrm{A}$ gets from the 
interconnection because $c_{B}^{\prime}-c_{B}-r=c_{A}^{\prime}-c_{A}+r$. Then, the payoff of $\mathrm{A}$ will be

$$
v_{A}=r-c_{A}=\frac{\left(c_{B}^{\prime}-c_{A}^{\prime}\right)-\left(c_{A}+c_{B}\right)}{2}=v_{A}^{\prime}+\frac{\Delta}{2}
$$

and the payoff of $\mathrm{B}$ will be

$$
v_{B}=-r-c_{B}=\frac{\left(c_{A}^{\prime}-c_{B}^{\prime}\right)-\left(c_{A}+c_{B}\right)}{2}=v_{B}^{\prime}+\frac{\Delta}{2}
$$

Note that $\left(v_{A}, v_{B}\right)$ is the Nash solution.

It is easy to see that the payoff of each $A S$ at the Nash solution $\left(v_{A}\right.$ and $\left.v_{B}\right)$ is higher than its payoff at the outside option ( $v_{A}^{\prime}$ and $v_{B}^{\prime}$, respectively) if and only if $\Delta>0$. So, with Nash-Peering both ASes are better off compared to the outside option, even if one of them has to pay the other.

If $\mathrm{A}$ benefits more from the interconnection than $\mathrm{B}$ $\left(c_{B}^{\prime}-c_{B}<c_{A}^{\prime}-c_{A}\right)$, the previous equations still hold but for a negative payment $r$, i.e., A would need to pay $-r$ to B to obtain the Nash solution.

The previous simple equations describe the proposed Nash-Peering interconnection framework. To summarize, $\mathrm{A}$ and $\mathrm{B}$ should agree to directly interconnect if and only if the surplus $\Delta$ is positive because in that case both of them will benefit. If $B$ benefits more from the interconnection than A then B should pay $r$ to A, where $r$ is given by (2); otherwise A should pay $-r$ to B. It is important to note that the Nash-Peering framework does not rely on any arbitrary conventions about the direction of payment; it does not assume that the sender of the traffic should always pay, for instance.

\subsection{Remarks}

The negotiation terms involve only the costs incurred by $\mathrm{A}$ and $\mathrm{B}$ when the interconnection is not in place $\left(c_{A}^{\prime}\right.$ and $\left.c_{B}^{\prime}\right)$ and when it is $\left(c_{A}\right.$ and $\left.c_{B}\right)$. These costs depend, in general, on the location of the interconnection. For example, if A is a US-based ISP and B is a European ISP, interconnecting in New York would introduce lower costs for A and higher costs for B than if they interconnect in Amsterdam. Similarly, the previous costs are route-dependent. For example, the cost of a transcontinental route is higher than the cost of a route that delivers the traffic in the same metro area. Or, the cost of a route that goes through a transit provider is higher than the cost of a local route.

The exposition so far assumed that A exports a route to $\mathrm{B}$ and the latter sends the traffic volume $T$ through A. In practice, the interconnection will carry traffic in both directions. If we apply the Nash-Peering framework separately to each direction of the interconnection, it could be that $A$ has to pay a fee $r_{A B}$ to $\mathrm{B}$ and $B$ has to pay a fee $r_{B A}$ to A. The two ASes can then combine the two payments into a single lump-sum payment.

Settlement-free peering is a special case of Nash-Peering when $c_{B}^{\prime}-c_{B}=c_{A}^{\prime}-c_{A}$, i.e., when the interconnection is equally beneficial to both ASes. Paid-peering, on the other hand, may seem initially as similar to NashPeering. This is not the case however for two reasons: first, in paid-peering it is always the sender (typically a content provider) that has to pay the receiver (typically an access provider), and second, the price associated with paid-peering may not be determined based on the interconnection costs for the two ASes and the costs of their outside options.

We should emphasize that Nash-Peering does not consider the economic value of the information that is carried by the traffic flow $T$ (e.g., whether the traffic is HBO premium content or whether it originates from Google). Considering that economic value and trying to somehow distribute it between the ASes that the traffic goes through is a highly controversial subject and is viewed by many as a violation of network neutrality [10]. Nash-Peering does not do that - it only considers the costs incurred by the two ASes in carrying this traffic through their infrastructure.

Equations (3) and (4) show that the utility of both ASes increases with the surplus $\Delta$. So, given a certain outside option with costs $c_{A}^{\prime}$ and $c_{B}^{\prime}$, both ASes have the same incentive: to decrease their internal interconnection costs $c_{A}$ and $c_{B}$ as much as possible. As long as $\left(c_{A}+c_{B}\right)$ is minimized, both $\mathrm{A}$ and $\mathrm{B}$ will split the corresponding maximized surplus equally; the relative magnitude of $c_{A}$ and $c_{B}$ does not matter.

What if the two ASes simplify their negotiations by considering many routes simultaneously, and averaging the underlying interconnection costs across all routes? ${ }^{2}$ This can be done in practice but it may result in loss of economic efficiency. To see why, consider a simple example with two routes. Suppose that the surplus for route- 1 is positive $\left(\Delta_{1}=\left(c_{A, 1}^{\prime}+c_{B, 1}^{\prime}\right)-\left(c_{A, 1}+c_{B, 1}\right)>0\right)$ but the surplus for route-2 is negative. If the two ASes negotiate for both routes at the same time, averaging the corresponding cost parameters, it may be that the aggregate surplus $\Delta$ is positive. In that case the interconnection will be established, even though both $\mathrm{A}$ and B would be better off if they had only interconnected for route-1. Similarly, if $\Delta<0$, they will not interconnect even though they would be better off if they had only considered route- 1 .

\section{DISPUTES AND NASH-PEERING}

In this section we briefly review some common types of interconnection disputes (see [1] for a recent historical analysis) and discuss how they would be resolved in the Nash-Peering framework.

\subsection{Access vs. Content providers}

$9^{2}$ Each route would be weighted based on the traffic volume it carries. 
Especially in the last few years, there are frequent disputes between Access Providers (APs) and Content Providers (CPs). The sf-Peering framework does not allow these two types of ASes to negotiate their interconnection in a principled manner based on actual costs and available alternatives. APs and CPs are very different in terms of their traffic patterns: the former mostly consume traffic while the latter mostly produce traffic, meaning that any peering conditions based on traffic ratios will obviously not be met. CPs often request sf-Peering with APs (so that they both minimize upstream transit fees) while APs claim that CPs benefit much more from such interconnections.

In the Nash-Peering framework, APs and CPs can always interconnect if there is a mutual benefit for both of them (positive surplus). For instance, in an actual recent case between a major US ISP (Comcast) and a major video provider (Netflix), the CP's outside option was to pay one or more transit providers (or CDNs) while the AP's outside option was to rely on one or more sf-Peering links with those transit providers, suggesting that $c_{C P}^{\prime} \gg c_{A P}^{\prime}$. Further, if the interconnection(s) between them would take place closer to the CP's data centers, it would also be that $c_{A P} \gg c_{C P}$. In a scenario like this, Nash-Peering specifies that the CP will have to pay the AP for the interconnection. This payment may be close to zero, or it may even be reversed (from the $\mathrm{AP}$ to the $\mathrm{CP}$ ), if the $\mathrm{CP}$ has its own backbone network that delivers the traffic very close to the final "eyeballs" (meaning that $c_{A P} \approx c_{C P}$ or even that $c_{A P}<c_{C P}$ ), while the outside option is equally expensive for both of them (perhaps they both use the same transit provider, with $\left.c_{C P}^{\prime} \approx c_{A P}^{\prime}\right)$.

\subsection{The fallacy of traffic ratios}

A second common dispute is between ISPs (transit or access providers) that have established sf-Peering interconnections based on a traffic ratio constraint $\gamma$ (i.e., A and $\mathrm{B}$ peer if the traffic between them, $T_{A B}$ and $T_{B A}$, satisfies the constraint $1 / \gamma<\frac{T_{A B}}{T_{B A}}<\gamma$, where $\gamma$ is typically between 2 and 5). This condition, however, does not have have any relevance to the economic benefits of this interconnection for each party. It is possible that the condition is not met, even though the interconnection's surplus is positive, meaning that the two ISPs would not establish an sf-Peering link even though a Nash-Peering interconnection would be beneficial for both of them. The opposite can also happen, if the surplus is negative but the traffic ratio constraint is met.

The traffic ratio condition has its roots in the economics of telephone networks where every long-distance call would require roughly the same resources from the two carriers. Nash-Peering replaces this constraint with economic considerations that do not make any assumptions about the correspondence between economic value or cost and the corresponding traffic flows.

\subsection{Tier-1 "peering wars"}

A third type of dispute arises due to the status of some ASes as "Tier-1" (meaning that they do not have any transit providers). In the current Internet, all Tier1 providers have to be interconnected with a full-mesh of sf-Peering relations. The presence of such a clique results in a problematic state where existing Tier- 1 providers adopt highly restrictive peering policies in order to avoid interconnecting with networks outside the clique (there is no economic incentive for a network in the clique to peer with a network outside the clique). Similarly, suppose that an AS $\mathrm{X}$ is a member of the Tier-1 clique but another AS Y claims that X no longer satisfies Y's peering criteria. If $\mathrm{Y}$ terminates the sf-Peering interconnection with $\mathrm{X}$, some ASes in the customer cone of $\mathrm{Y}$ would be disconnected from some ASes in the customer cone of $\mathrm{X}$. This risk of network partitioning causes some ASes to retain existing peering links even if they are no longer beneficial to them.

In other words, the clique of Tier-1 providers is hard to evolve and its static structure can cause disputes between incumbents and newcomers. In the Nash-Peering framework, Tier-1 providers do not have any special privilege (other than not having an upstream transit provider). All their interconnections can be based on Nash-Peering, instead of sf-Peering, and there would be dynamic exchanges of money between them that change in terms of amount and direction from month to month. Further, a new entrant does not need to negotiate rigid and restrictive peering policies, but can peer with any member of the clique (possibly with a payment) as long as it is mutually beneficial to do so.

\subsection{Not all routes cost the same}

Finally, we believe that the current interconnection framework does not provide the right incentives for ISPS to invest in high-cost routes that reach remote or sparsely populated destinations such as rural regions. The main issue is that the same transit price is typically applied on all routes, independent of the cost associated with each route. At the same time, intense competition forces all transit providers to reduce their transit prices as much as possible. So, an ISP does not have the incentive to invest in the infrastructure that a high-cost route requires, given that that route will not generate additional revenue.

With Nash-Peering, on the other hand, the interconnection fees are determined based on the cost of a route. If the $\operatorname{cost} c_{A}$ of the route that $\mathrm{A}$ advertises to $B$ increases, while the three other parameters of the interconnection $\left(c_{A}^{\prime}, c_{B}^{\prime}, c_{B}\right)$ are constant, the payment $r$ from $\mathrm{B}$ to $\mathrm{A}$ will also increase (as long as $\Delta>0$ ). This gives $\mathrm{A}$ the incentive to invest in high-cost routes that 
would be under-provisioned today.

\section{NASH-PEERING IN PRACTICE}

So far, our exposition assumed that A and B will agree to adopt the Nash solution, without considering the actual bargaining process between them or the information they will need during that process. Here, we explain how we envision Nash-Peering in a more practical context.

\subsection{The bargaining process}

A bargaining process between two rational players, when modeled as a sequential game, converges to a unique subgame-perfect equilibrium that is identical to the Nash solution [3]. The mathematical assumptions for this result (see Proposition-3) are reasonable and we expect them to be true in practice (see Assumptions 1, 2, 8 and 9). The key condition is that the probability that the negotiations between $\mathrm{A}$ and $\mathrm{B}$ will fail, even though the surplus $\Delta$ is positive, is the same for both players and negligible. In other words, both players negotiate patiently and rationally, knowing that if they manage to reach agreement they will mutually benefit compared to their outside option. Considering impatient or asymmetric players (e.g., A is an AS that desperately needs this interconnection, while $\mathrm{B}$ does not care much about it) is an important question for future research but outside the scope of this paper.

Based on the previous result of [3], we can assume that the two ASes A and B will either directly agree to adopt the Nash solution, as a normative statement about how they should be sharing the costs of their interconnection, or it is reasonable to expect that a bargaining process between them will eventually result in the Nash solution.

\subsection{Interconnection parameters}

Even if A and B agree to adopt the Nash solution, what information will they need to have access to? The Nash solution requires knowledge of $c_{A}, c_{B}, c_{A}^{\prime}$, and $c_{B}^{\prime}$. We refer to these four terms as the interconnection parameters: they represent the costs that are associated with this traffic volume in the outside option $\left(c_{A}^{\prime}, c_{B}^{\prime}\right)$, and with the corresponding costs if that interconection is actually established $\left(c_{A}, c_{B}\right)$.

First, an AS should be able to estimate the interconnection parameters for its own traffic. The more challenging question is how to estimate the corresponding parameters of the other AS. Obviously, A should not just trust $B$ when the latter claims that its relevant parameters are $c_{B}$ and $c_{B}^{\prime}$. Later in this section, we describe an one-sided estimation process that would allow A to estimate the parameters $c_{B}$ and $c_{B}^{\prime}$ of $\mathrm{AS}$ $B$, at least approximately. More generally, however, we do not believe that these negotiations have to be based entirely on one-sided estimated parameters. We envision that when two ASes negotiate their interconnection agreement they will be presenting quantitative cost analyses to each other for the traffic they will be exchanging, allowing the other AS to verify claims about the cost of the outside option or of the negotiated interconnection.

\subsection{The granularity of negotiations}

In practice, the negotiations between $\mathrm{A}$ and $\mathrm{B}$ will not need to consider each BGP route separately. First, even though there are about half a million BGP routes today, the vast majority of the traffic that is directly exchanged between two ASes is dominated by a relatively small number of BGP routes [17]. For instance, if A is Comcast and B is Google, the two ASes could focus on the rather small number of routes that originate from Comcast's network, given that the vast majority of the traffic in this interconnection would flow from Google data centers to Comcast subscribers.

Second, we expect that the interconnection parameters of large groups of routes will be roughly identical, when considering a specific location. For instance, if $\mathrm{A}$ is Cogent and B is Deutsche Telekom, and they consider interconnecting in Frankfurt, most Cogent routes for US-based destination networks would probably be grouped together. On the other hand, Cogent routes for Asia-based destination networks would probably be associated with different interconnection parameters, and they would be negotiated as a different group of routes. Also, these parameters would be different when Cogent and Deutsche Telekom interconnect in Ashford VA, instead of Frankfurt.

A quantitative analysis of how many groups of routes would need to be negotiated separately, at a given interconnection location and for a given pair of ASes, is certainly an important question that we will investigate in future work. It is clear though that negotiating large bundles of routes with similar interconnection parameters will significantly decrease the accounting complexity of Nash-Peering.

\subsection{One-sided parameter estimation}

We assume that a network with complete knowledge of its internal infrastructure can estimate its own internal costs (interconnect and backhaul), perhaps as proposed by Motiwala et al. [21].

An AS also needs to estimate the internal costs and outside options of its potential peers. To estimate the outside option of B, the first option is for A to use BGP and traceroute measurements from a route server in B's network, along with AS-relationship data [5], to infer whether B's path toward destination D is via a customer, provider, or peer. A second option is to use $A S$ customer cone data [5] to infer whether B could reach 
prefix D using either customer or peer links. If B's outside option is a provider, A can estimate B's transit cost using available transit pricing data.

To estimate B's internal costs, A needs to know the ingress and egress points into B's network for the traffic toward destination D. A can use traceroutes from looking glass servers in B (or networks in B's customer cone), or the reverse traceroute system [16], to measure the path towards D, and hence infer the ingress and egress points into B's network. Assuming symmetry of cost factors, A can approximate B's internal costs using its own cost model and the ingress/egress points into B's network.

\section{RELATED WORK}

Dhamdhere et al. [9] proposed a value-based peering scheme that treats each interconnection as a paidpeering link, where a payment is exchanged depending on the "added value" that the interconnection offers to each party. Courcoubetis et al. [8] have also applied the theory of Nash bargaining to derive paid-peering prices (specifically targeting the negotiation between content and access providers) considering factors such as advertising revenues, subscriber loyalty, and interconnection or capacity costs. Jahn and Prüfer [15] analyzed a model in which two ISPs with asymmetric sizes compete for subscribers while at the same time they consider paid-peering, based on Nash bargaining, as a way to interconnect. They assume equal internal costs for both ISPs and a variable transit fee charged by a single transit provider. Besen et al. [2] analyzed the outcome of Nash bargaining between two peers in the absence of an outside option, which incurs service degradation, and loss of customers and revenue. Nash-Peering differs from these earlier models in that the interconnection fees are determined based on costs rather than the economic "value" of a flow (which is much harder to measure - and rather controversial).

Valancius et al. [26] demonstrated that ISPs could maximize transit profits by employing a tiered pricing scheme, charging different prices for different routes they also showed that a small number of tiers would be sufficient. Their scheme focuses on transit relations, however, and does not involve price bargaining. MINT [25] and Route Bazaar [4] are two schemes where "route sellers" advertise connectivity for different path segments and "route buyers" compose end-to-end paths by purchasing connectivity over specific path segments. Route Bazaar and MINT do not focus on the economics of how the prices are set, or how prices relate to costs and outside options. Tan et al. [24] showed how providers can establish interconnection agreements to dynamically trade network capacity. This demonstrates how "paid peering" can be employed to improve network utilization and create incentives to improve infrastructure.
Prior research by Ma et al. [19, 20] employs cooperative game theory to model multilateral interconnections betweeen different types of networks in the Internet ecosystem. They proposed the use of the Shapley Value to achieve fair and stable division of surplus among different types of providers. Nash-Peering differs from that approach in that it only requires bilateral contracts between ASes. Multilateral interconnection agreements are much harder to establish and manage in practice.

From a policy standpoint, Faratin et al. [11] discuss the emergence of paid peering, highlight the increased complexity of the negotiations involved in peering, and discuss the implications for telecommunications policy. Clark et al. [6] analyzed the reasons why settlement-free peering based on traffic ratios could make way for paidpeering between CDNs and access ISPs. The authors advocated increased transparency into costs, traffic patterns, and interconnection terms over regulation of interconnection. Economides and Tåg [10] focus on network neutrality regulation and demonstrate the effects of regulation on the total surplus of peering relations.

\section{DISCUSSION}

Nash-Peering represents a major departure from the current interconnection framework. Instead of the limiting dichotomy of AS relations into transit and sf-Peering, it offers a more general, efficient and fair framework in which two ASes evaluate the economic benefits that an interconnection would provide them, at the level of groups of routes with similar costs, and split any potential surplus equally among them.

The next step in this research will be to examine the Internet-wide effects of bilateral Nash-Peering interconnections. For instance, a change in the interconnection between $\mathrm{A}$ and $\mathrm{B}$ may trigger changes in many interdomain routes. The latter may affect the traffic volume in other interconnections, between different pairs of ASes, i.e., these bilateral interconnections have network-wide externalities. Of course such externalities are also present in the current interconnection framework but Nash-Peering will probably introduce more complex dynamics due to the finer granularity of bilateral negotiations. Interestingly, the network externalities of bilateral contracts is also an active research area in economic theory, and we plan to leverage that emerging body of knowledge [13]. 


\section{REFERENCES}

[1] S. Bafna, A. Pandey, and K. Verma. Anatomy of Internet Peering Disputes. CoRR, abs/1409.6526, 2014.

[2] S. Besen, P. Milgrom, B. Mitchell, and P. Srinagesh. Advances in routing technologies and Internet peering agreements. The American Economic Review, 91(2):292-296, 2001.

[3] K. Binmore, A. Rubinstein, and A. Wolinsky. The Nash bargaining solution in economic modelling. The RAND Journal of Economics, pages 176-188, 1986.

[4] I. Castro, A. Panda, B. Raghavan, S. Shenker, and S. Gorinsky. Route Bazaar: Automatic Interdomain Contract Negotiation. In Proceedings of the 15th USENIX Conference on Hot Topics in Operating Systems, HOTOS'15. USENIX Association, 2015.

[5] Center for Applied Internet Data Analysis. CAIDA - AS Rank. http://as-rank. caida.org.

[6] D. Clark, W. Lehr, and S. Bauer. Interconnection in the Intenret: The Policy Challenge. In Telecommunications Policy Research Conference (TPRC), Aug 2011.

[7] D. D. Clark, S. Bauer, W. Lehr, K. Claffy, A. D. Dhamdhere, B. Huffaker, and M. Luckie. Measurement and analysis of Internet interconnection and congestion. In $2014 T P R C$ Conference Paper, 2014.

[8] C. Courcoubetis, L. Gyarmati, N. Laoutaris, P. Rodriguez, and K. Sdrolias. Negotiating premium peering prices: A quantitative model with applications. ACM Trans. Internet Technol., 16(2):14:1-14:22, Apr. 2016.

[9] A. Dhamdhere, C. Dovrolis, and P. Francois. A Value-based Framework for Internet Peering Agreements. In International Teletraffic Congress (ITC), Amsterdam, The Netherlands, Oct 2010.

[10] N. Economides and J. Tåg. Network neutrality on the Internet: A two-sided market analysis. Information Economics and Policy, 24(2):91-104, 2012.

[11] P. Faratin, D. Clark, S. Bauer, W. Lehr, P. Gilmore, and A. Berger. The Growing Complexity of Internet Interconnection. Communications \& Strategies, 1(72):51-72, 2008.

[12] Federal Communications Commission. Open Internet Order, 2015.

[13] C. C. Fontenay and J. S. Gans. Bilateral bargaining with externalities. The Journal of Industrial Economics, 62(4):756-788, 2014.

[14] G. Huston. ISP Survival Guide: Strategies for Running a Competitive ISP. John Wiley \& Sons, Inc., New York, NY, USA, 1999.

[15] E. Jahn and J. Prüfer. Interconnection and competition among asymmetric networks in the Internet backbone market. Information Economics and Policy, 20(3):243-256, 2008.

[16] E. Katz-Bassett, H. V. Madhyastha, V. K. Adhikari, C. Scott, J. Sherry, P. Van Wesep, T. Anderson, and A. Krishnamurthy. Reverse traceroute. In Proceedings of the 7th USENIX Conference on Networked Systems Design and Implementation, NSDI'10, pages 15-15. USENIX Association, 2010.

[17] C. Labovitz, S. Iekel-Johnson, D. McPherson, J. Oberheide, and F. Jahanian. Internet inter-domain traffic. ACM SIGCOMM Computer Communication Review, 41(4):75-86, 2011.

[18] A. Lodhi, N. Larson, A. Dhamdhere, C. Dovrolis, and k. claffy. Using peeringDB to Understand the Peering Ecosystem. SIGCOMM Comput. Commun. Rev., 44(2):20-27, Apr. 2014.

[19] R. T. B. Ma, D. M. Chiu, J. C. S. Lui, V. Misra, and D. Rubenstein. Internet Economics: The Use of Shapley Value for ISP Settlement. IEEE/ACM Trans. Netw., 18(3):775-787, June 2010.

[20] R. T. B. Ma, D. M. Chiu, J. C. S. Lui, V. Misra, and D. Rubenstein. On Cooperative Settlement Between Content, Transit, and Eyeball Internet Service Providers. IEEE/ACM Trans. Netw., 19(3):802-815, June 2011.

[21] M. Motiwala, A. Dhamdhere, N. Feamster, and A. Lakhina. Towards a cost model for network traffic. SIGCOMM Comput. Commun. Rev., 42(1):54-60, Jan. 2012.

[22] J. F. Nash Jr. The bargaining problem. Econometrica: Journal of the Econometric Society, pages 155-162, 1950.

[23] J. F. Nash Jr. Two-person cooperative games. Econometrica: Journal of the Econometric Society, pages 128-140, 1953.

[24] Y. Tan, I. R. Chiang, and V. S. Mookerjee. An economic analysis of interconnection arrangements between Internet backbone providers. Operations research, 54(4):776-788, 2006.

[25] V. Valancius, N. Feamster, R. Johari, and V. Vazirani. MINT: A Market for INternet Transit. In Proceedings of the 2008 ACM CoNEXT Conference, CoNEXT '08, pages 70:1-70:6. ACM, 2008.

[26] V. Valancius, C. Lumezanu, N. Feamster, R. Johari, and V. V. Vazirani. How many tiers?: Pricing in the internet transit market. In Proceedings of the ACM SIGCOMM 2011 Conference, SIGCOMM '11, pages 194-205. ACM, 2011.

[27] William B. Norton. The Internet Peering Playbook: Connecting to the Core of the Internet. DrPeering Press, 2012. 